\section{NSCLC: Atezolizumab mit Überlebensvorteil unabhängig vom PD-L1-Status}

Rittmeyer A et al. Atezolizumab versus docetaxel in patients with previously treated nonsmall-cell lung cancer (OAK): a phase 3, openlabel, multicentre randomised controlled trial. Lancet 2017; 389: 255 - 265

Indem der Checkpoint-Inhibitor Atezolizumab an das Protein PD-L1 (Programmed Death Ligand-1) bindet und so die Interaktion von PD-L1 und PD-1 blockiert, fördert der Antikörper wahrscheinlich die T-Zell-Aktivierung. In der ersten Phase-3-Studie (OAK) mit Atezolizumab konnten nun Achim Rittmeyer et al. zeigen, dass sich beim NSCLC das Gesamtüberleben gegenüber Docetaxel deutlich verlängern lässt.

In die Studie wurden Patienten mit lokal fortgeschrittenem oder metastasiertem NSCLC unabhängig vom PD-L1-Status aufgenommen, die bereits $1-2$ Chemotherapien erhalten hatten. Mindestens eine der Chemotherapien musste platinbasiert gewesen sein. Die internationale Arbeitsgruppe stratifizierte die Teilnehmer entsprechend der PD-L1-Expression, der Zahl vorheriger Chemotherapien und der Histologie. Insgesamt 1225 Patienten erhielten randomisiert alle 3 Wochen entweder $1200 \mathrm{mg}$ Atezolizumab i.v. oder $75 \mathrm{mg} / \mathrm{m}^{2}$ Docetaxel i.v. solange, bis inakzeptable Toxozitäten oder Krankheitsprogression auftraten. Die Tumor- beurteilungen erfolgten bis Woche 36 alle 6 Wochen und anschließend alle 9 Wochen. Das Gesamtüberleben in der Intention-to-Treat-Population (ITT) und bei Patienten mit einer PD-L1-Expression $\geq 1 \%$ in Tumorzellen (TC) oder tumorinfiltrierenden Zellen (IC) bildete den primären Endpunkt. In die primäre Wirksamkeitsanalyse gingen die Ergebnisse von jeweils 425 Patienten der beiden Behandlungsarme ein, die sekundäre Wirksamkeitsanalyse basierte auf der Gesamtpopulation.

\section{Ergebnisse}

Die 63-64 Jahre alten Patienten hatte vor der OAK-Studie zu 75\% eine und zu $25 \%$ zwei Therapien erhielten. Bei $74 \%$ handelte es sich um ein Nichtplattenepithelkarzinom und bei $26 \%$ um ein Plattenepithelkarzinom. Unter Atezolizumab lag die Behandlungsdauer median bei 3,4 Monaten und unter Docetaxel bei 2,1 Monaten. Das mediane Gesamtüberleben (ITT-Population) erreichte in der Atezolizumab-Gruppe 13,8 Monate und in der Docetaxel-Gruppe 9,6 Monate (Hazard Ratio [HR] 0,73, $95 \%$ Konfidenzintervall $[\mathrm{KI}]$ 0,62-0,87, $\mathrm{p}=0,0003)$. Bei Patienten mit PD-L1-Expression verlängerte sich das Gesamtüberleben von median 10,3 (Docetaxel) auf 15,7 Monate (Atezolizumab) (HR 0,74, 95\% KI 0,58$0,93, p=0,0102)$. Davon profitierten am stärksten Patienten mit der höchsten PD-L1-Expression (Atezolizumab: 20,5 Monate vs. Docetaxel: 8,9 Monate), aber auch statistisch signifikant Patienten ohne PD-L1-Expression (Atezolizumab: 12,6 Monate vs. Docetaxel: 8,9 Monate).
Während in der ITT-Population das progressionsfreie Überleben keine signifikanten Unterschiede ergab (Atezolizumab: 2,8 Monate vs. Docetaxel: 4,0 Monate) war die Dauer des Respons in der Atezolizumab-Gruppe deutlich länger (16,3 Monate vs. 6,2 Monate). Unerwünschte Wirkungen mit Grad 3-4 traten zu $15 \%$ in der PD-L1-AntikörperGruppe und zu $43 \%$ in der Taxan-Gruppe auf. Zu Therapieabbrüchen aufgrund unerwünschter Ereignisse kam es bei $8 \%$ (Atezolizumab) bzw. 19\% (Docetaxel) der Patienten.

FAZIT

Die Studienergebnisse belegen, dass mit dem PD-L1-Antikörper Atezolizumab bei vorbehandelten NSCLC-Patienten gegenüber Docetaxel ein klinisch bedeutender Überlebensvorteil erreicht werden kann. Dieser Effekt ist unabhängig von der PD-L1-Expression.

Matthias Manych, Berlin 